\title{
Remote Control using Voice Recognition based on Arduino
}

\author{
Jinan N. Shehab \\ Department of Communication Engineering, College of Engineering, University of Daiyla \\ engjinan83@gmail.com
}

\begin{abstract}
Home automation becomes important, because it gives the user convenient and easy method to use home appliances. This paper aims to help people with special needs or physical disabilities and injuries by paralysis to control any device using infrared technology using voice commands based on the voice recognition system (voice recognition unit V3) system can recognize voice commands, convert them to desired data coordination and data transmission via IR transmitter and microcontroller (Arduino Uno) Receiving this signal by IR sensor to control TV receiver then get a full remote control that works by voice commands. The software consists of a Micro C language programmable microcontroller. This system is of low cost and flexible with growing variety of devices that can be controlled.
\end{abstract}

Keywords: Arduino Uno 328, Voice controlled remote, Voice recognition module V3, TV remote control.

Paper History: (Received: 18/12/2017; Accepted: 24/5/2018)

\section{Introduction}

Demography in the world shows that the population is rising rapidly, which in turn increases people's average life expectancy as well as the many accidents that cause the loss of limbs in the human body. In today's era, one of the major growing industries is home automation because we have tight time as well as we always look to change the way we live, and this home automation system is not a new phenomenon but it is old. While the only difference is that elderly and physically disabled people are dealing with luxury, sophisticated and expensive home automation platforms [1]. The aim of this project is to provide service to those older, disabled. So that by giving the command through the voice, they can control devices such as light, fan, heater, TV, etc. at home. The other goal of this project is to make a system that is reasonably cheap, easy to operate or configure and easy to operate. There are various commercial and research projects available in the market on smart homes and voice recognition system. This work has six section includes the introduction, previous works, working equipment like IR sensors, voice recognition and the Arduino, the proposed system and finally result with discussion.

\section{Related Work}

In [2], suggested to design and implement a voice commands from the user to control robot name ROBOTVOICE. This work includes the macros software for voice named Easy VR Commander and Arduino micro-controller to control signals and Lab VIEW work with Bluetooth interface to remove the need to use buttons, dials and switches, consumers can easily operate appliances with their hands full or while doing other tasks.

In [3], proposed to use smartphones to control all application in the user home by their voice depending on an Arduino micro-controller. The system circuit involves an Arduino Uno microcontroller, which was used to process the speech commands from user and control the switching of devices. The Arduino and smartphone are connected by using Bluetooth application, also the system can use a widespread wireless technology to share data.

In [4], proposed design and implementation of the voice command to control the movements of the wheel chair and this work is very important for the physically handicapped person where the speech command is used to control the wheel chair. In this system Voice Recognition Kit (HM2007 Module) was used to recognize the speech command.

In [5], suggested to control two of the most important human uses the process of extinguishing and operation of light and fan depending on ATMEGA-328P and Android OS speech recognition. This work is an alternative and suitable way to operate devices based on sound and thus it helps people with special needs to exercise their lives like the average.

In [6], proposed a system which is developed with MATLAB-Arduino interface to control simple home appliances. Webcam is interfaced and accessed through MATLAB to have continuous monitoring of whole system setup. The GUI designed in MATLAB allows user to turn ON and OFF interfaced devices and shows current status of these devices in edit box.

In this work, proposed to design and implementation control system safe and easily operated, with low cost and efficiency aims to help people with special needs or physical disabilities and injuries by paralysis to control TV remote using voice commands. The prototype of this system, which is composed of two-parts transmitter and receiver, the major difference from the previous works lies in the use of the voice recognition V3, which allows to control 7 voices at a time in addition to receiving voice commands directly from the user through the microphone. 


\section{General Description of Equipment}

The equipment's that are used in this work are:

\section{ARDUINO}

It's an open-source physical measure demonstration based on a directly microcontroller board includes an ATmega328 microcontroller and an improvement environment for writing software for the board. Arduino is used to connect and communicate between the various devices. It contains a set of inputs such as sensors, power supply, switches and outputs that control the various devices such as lighting. Projects that rely on the Arduino can be accomplished by relying on it only or by linking it to the computer through different programs $[2,7,8]$.

Arduino Uno is microcontroller board and based on the Atmel's ATmega328 microcontroller. "Uno" means one in Italian and the uno board is the latest in a series of USB (Universal Serial Bus) .The Arduino Uno board has a $16 \mathrm{MHz}$ ceramic resonator, a USB connection, a power jack, an ICSP header, a reset button, 6 analog inputs and 14 digital input/output pins (of which 6 can be used as PWM outputs). The board has $32 \mathrm{~KB}$ flash memory of which $0.5 \mathrm{~KB}$ is used by boot-loader, $2 \mathrm{~KB}$ of SRAM, 1 $\mathrm{KB}$ of EEPROM and $16 \mathrm{MHz}$ clock speed. Arduino Uno board is shown in Figure $1[5,6]$

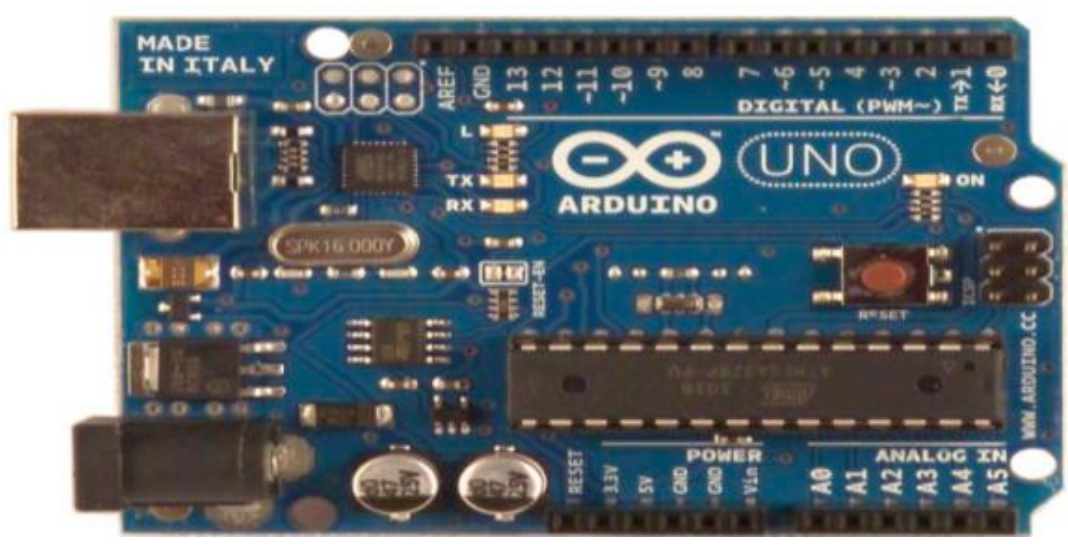

Figure 1: Arduino Uno Board

\section{Voice Recognition Module V3}

Voice Recognition Module is a simple unified control console. This produces a speaker-based voice recognition unit. Backup to 80 voice commands at all. Max 7 voice commands, all these commands are running at the same time. Any sound could be trained as command. Users need to train the module first before let it recognize any voice command. This board has 2 controlling ways: Serial
Port (full function), General Input Pins (part of function). General Output Pins on the board could generate several kinds of waves while corresponding voice command was recognized. On V3, voice commands are stored in one large group like a library. Any 7 voice commands in the library could be imported into recognizer. It means 7 commands are effective at the same time the Voice Recognition Module V3 is shown in Figure $2[9,10]$.

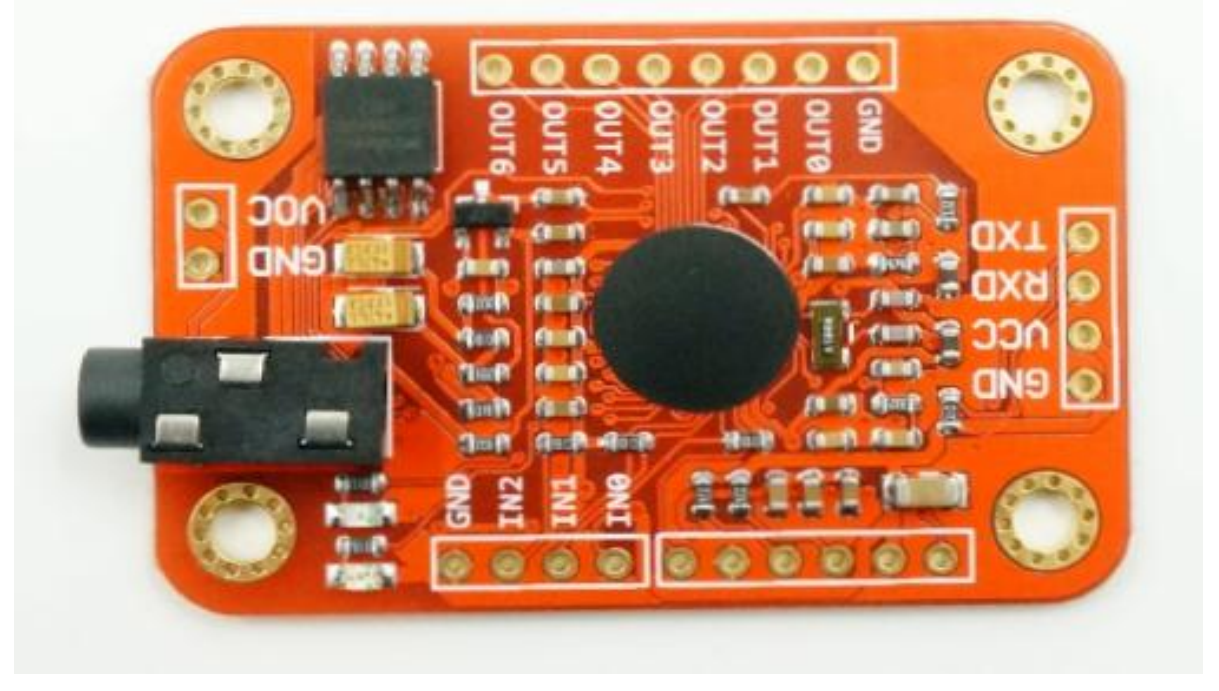

Figure 2: Voice recognition module V3 


\section{Infrared Sensor light-emitting diode (IR LED)}

IR, or infrared is a joint, cheap, and simple to employ wireless communication technology. The source of IR is about all place of us. The sun, light bulbs, or anything with heat is very bright in the IR spectrum. IR light is very identical to visible light, except the wavelength for IR is longer. This will attend to make it ideal for wireless communication and it is invisible to the human eye. IR
LED is used to transmit information to TV by using TV remote. IR LED is a two-lead semiconductor light source. It is a $\mathrm{p}-\mathrm{n}$ junction diode as shown in Figure 3 will emitting light when it is activated. When an appropriate voltage is supply to the leads, the electrons can recombine with electron holes within the device, emission energy in the form of photons. This action is called electroluminescence, and the color of the light is specified by the energy band gap of the semiconductor $[11,12]$.

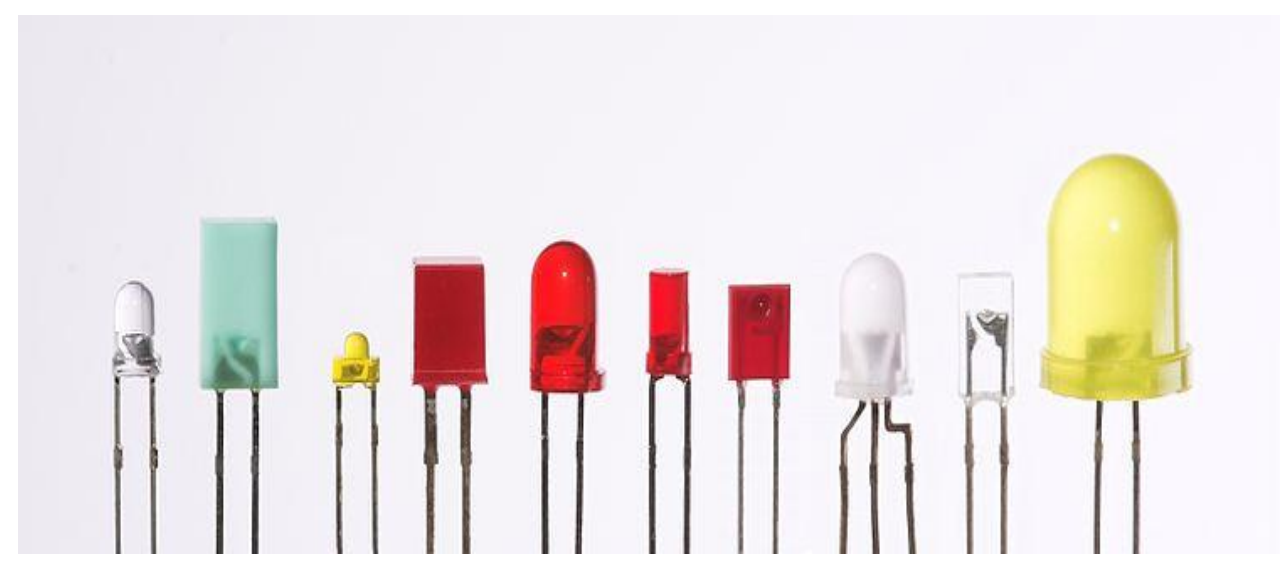

Figure 3: Different types from infrared sensor light-emitting diode (IR LED)

\section{Infrared Sensor (IR) Communication}

An Infrared sensor (PIR sensor) is an electronic device that measures infrared (IR) light emitted from remote control, this means the transmitter and receiver both in the same room because the communication from this type is usually used to connect the devices in short range, then this wireless communication desires optical visibility, means both the transmitter and the receiver must be in direct line of sight (L.O.S) [5].

\section{Proposed System}

The proposed system in this case consists of sensors, transmitter and receiver as shown in Figure 4 and Figure 5
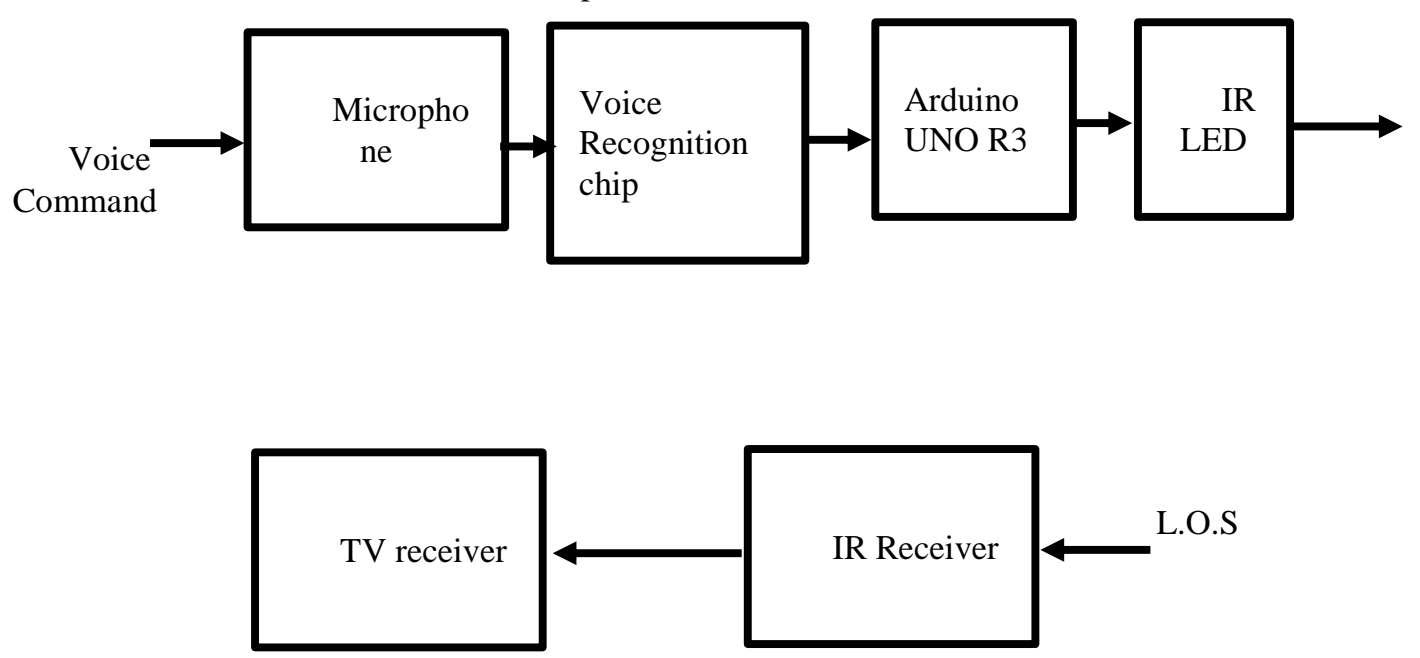

Figure 4: Block Diagram of Human Voice Controlled Remote based on Microcontroller 


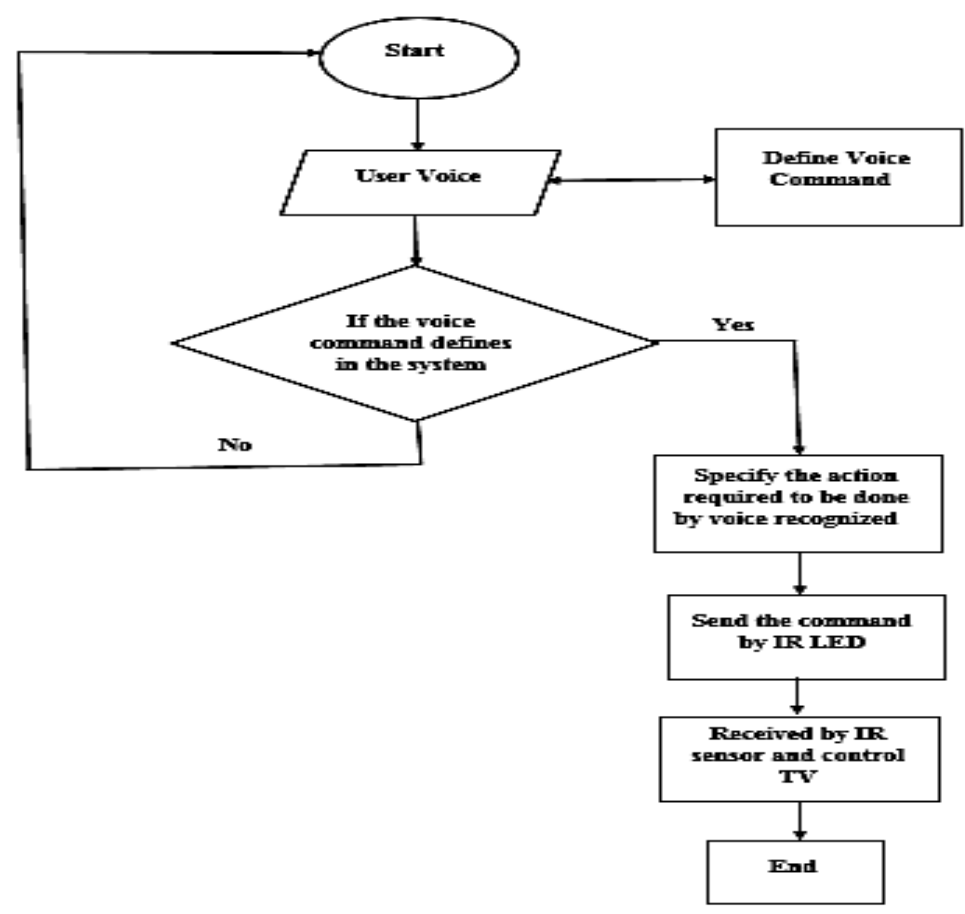

Figure 5: Flowchart of Voice Controlled Remote based on Microcontroller

The proposed system is combined with the software and hardware manipulations resulting into two sections; transmitter section and receiver section which gives the final model for the Home Automation System (HAS). As shown in figure. 4 and 5 , the required voice commands are stored in the voice recoginitionV3 then the user gives the voice command from microphone to be compared to the commands that were previously stored, if the command is truth, the speech signal is compared with the previously stored trained voice samples. Upon successful recognition of voice command, the Arduino microcontroller actuates the corresponding electrical device like turning on lights, and adjusting bed elevation. The data from the voice recognition is processed in Arduino controller and based on a set point value the automatic control action is taken to control the TV receiver depending on IR LED send signal to the IR receiver. The library out of 80 only 7 commands can be loaded into recognizer for the recognition process. Thus only 7 commands are effective at a time and to add another 7 commands recognizer needs to be cleared first. The module has two ways of controlling Serial Port, General Input Pins. General Output Pins on the board could generate several kinds of waves while corresponding voice command was recognized. Module has a recognition accuracy of $99 \%$ under ideal conditions.

\section{Result and Discussion}

\section{Voice Command Controlled Remote Control}

The system of two units: the transmitter unit (microphone with voice recognition, microcontroller, IR led) and the receiver unit IR sensor.

The microphone is used to give voice commands as input to control TV receiver. Mic which converts the voice signal to the electric signal and the signal is given to the voice recognition module. The voice recognition module converts the analog signal into digital signal and the signal is transferred to the pic microcontroller. The Microcontroller (Arduino) will send the IR signal to the receiver (TV receiver) by the IR transmitter (IR led) connected to the device and received this signal by IR sensor to control TV receiver according voice command storing in the microcontroller; the voice remote control consists of Arduino board and voice recognition module (V3) and IR transmitter, led, mic, battery. placed on a bread board as shown below in Figure 6 . 


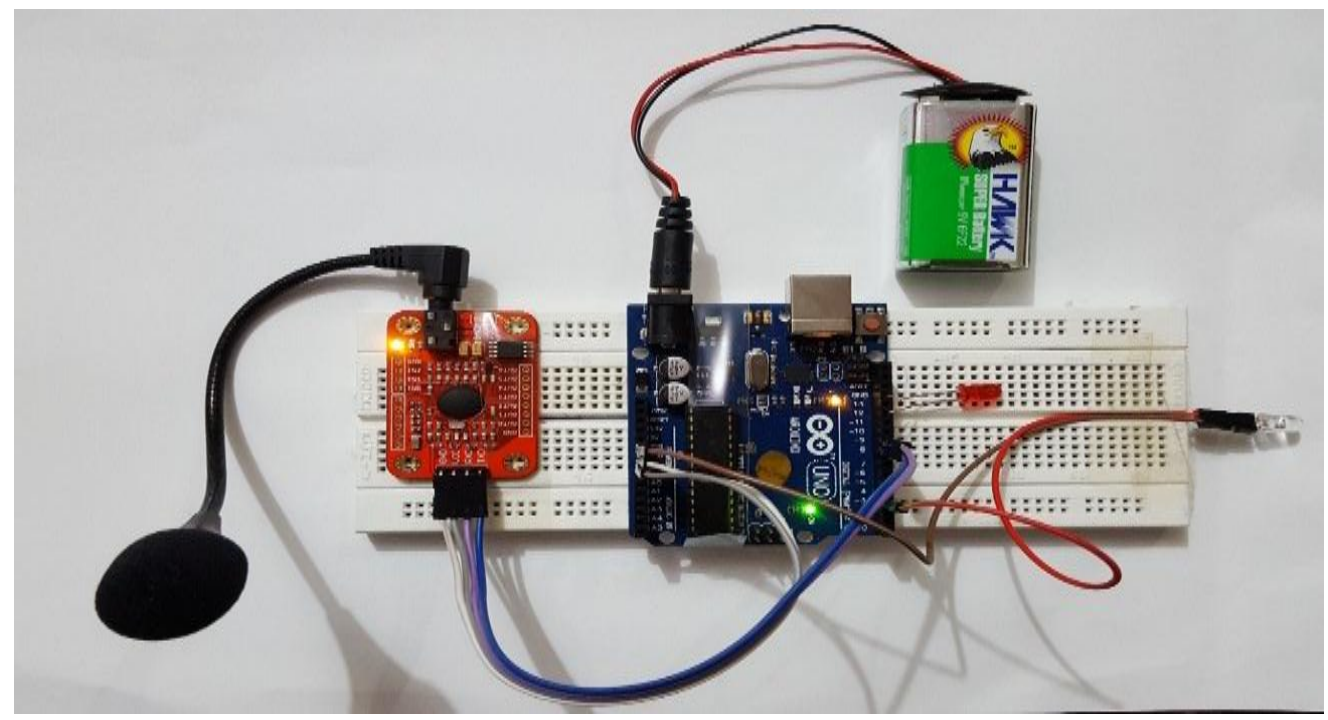

Figure 6: Hardware of Voice Controlled Remote based on Microcontroller

\section{Voice Command}

First, we record the voice commands that we want to use in the voice recognition module using Arduino IDE as shown in Figure.7. The voice commands used in this project as shown in the Table 1. After you have finished storing the voice commands in the voice recognition and connecting all the circuit parts; we are running it; the results of the work are shown in Figure $8,9,10$ :

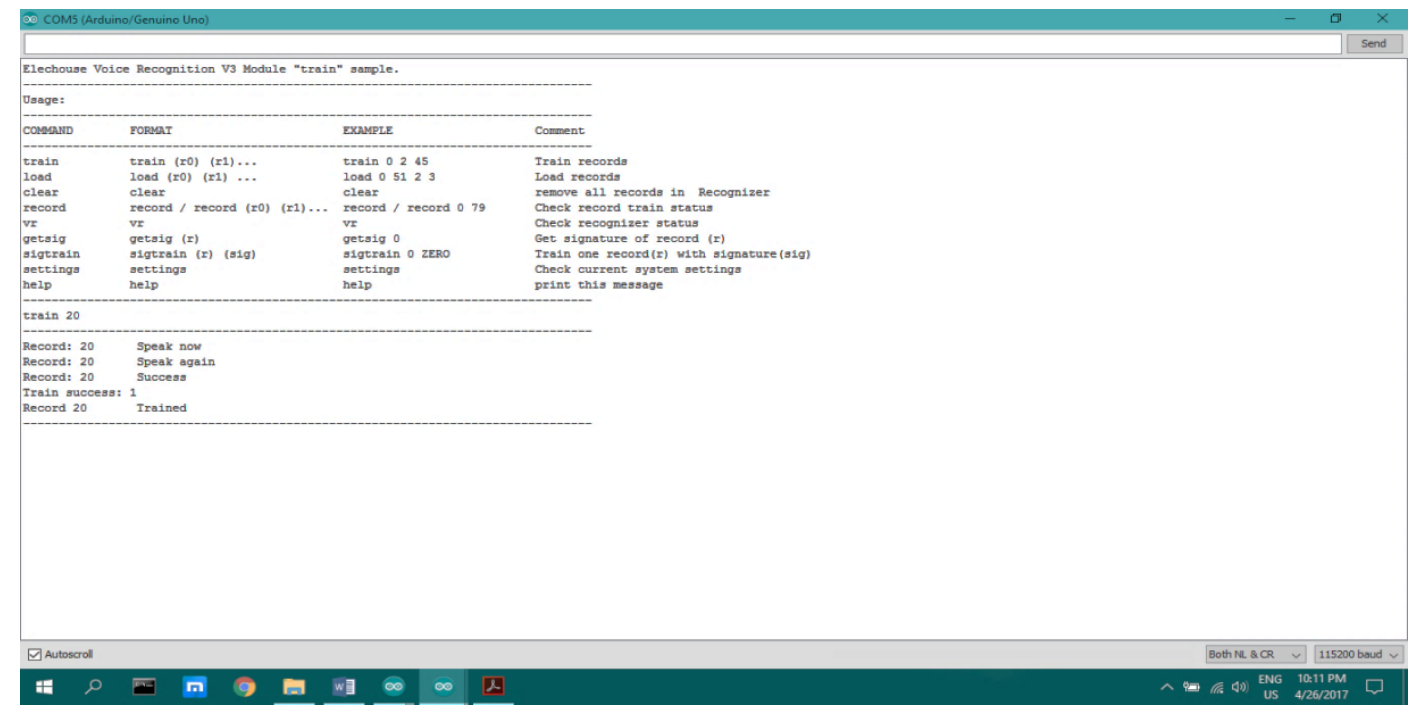

Figure 7: The voice recognition commands using Arduino IDE

Table 1 Voice Commands.

\begin{tabular}{l|l}
\hline Voice Command & Conditions \\
\hline On & Turn on the TV. \\
\hline Off & Turn off the TV. \\
\hline Up & Channel change (up). \\
\hline Down & Channel change (down). \\
\hline Mute & Mute the TV. \\
\hline Menu & Show list of contents in the TV. \\
\hline Exit & Exit the content list. \\
\hline Voi+ & Raise the sound \\
\hline Voi- & Decrease the sound \\
\hline
\end{tabular}




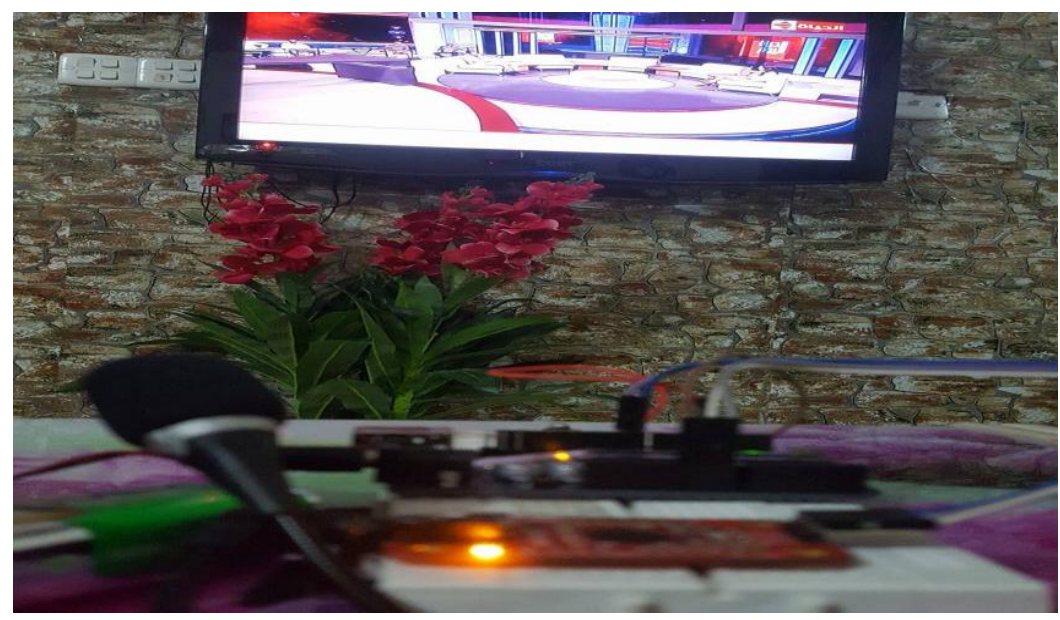

Figure 8: Testing of Turn ON the television

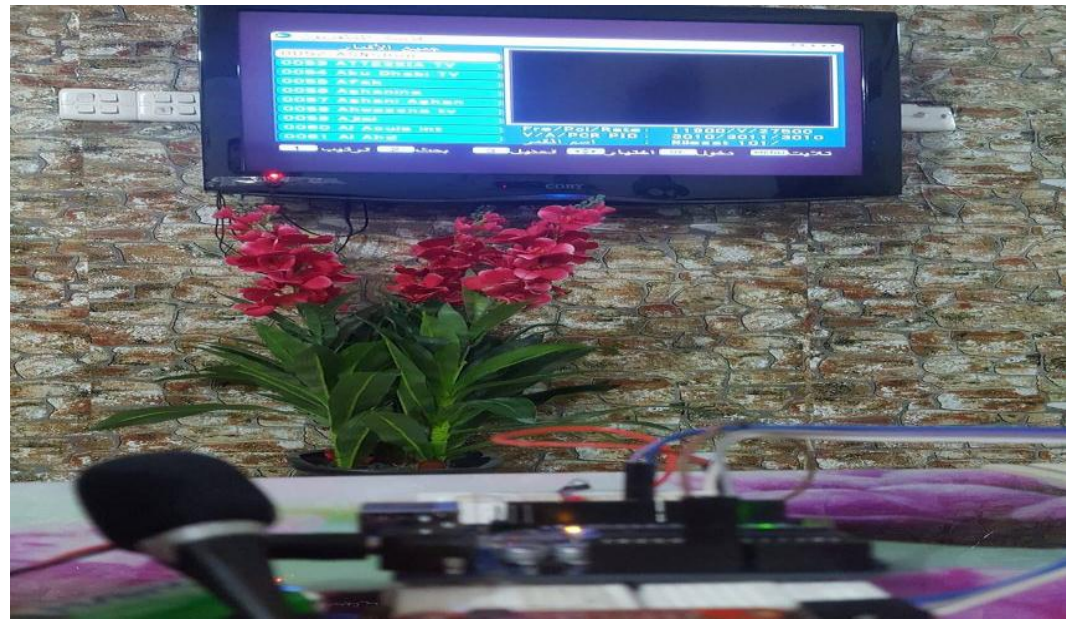

Figure 9: Testing of show list of contents in the TV

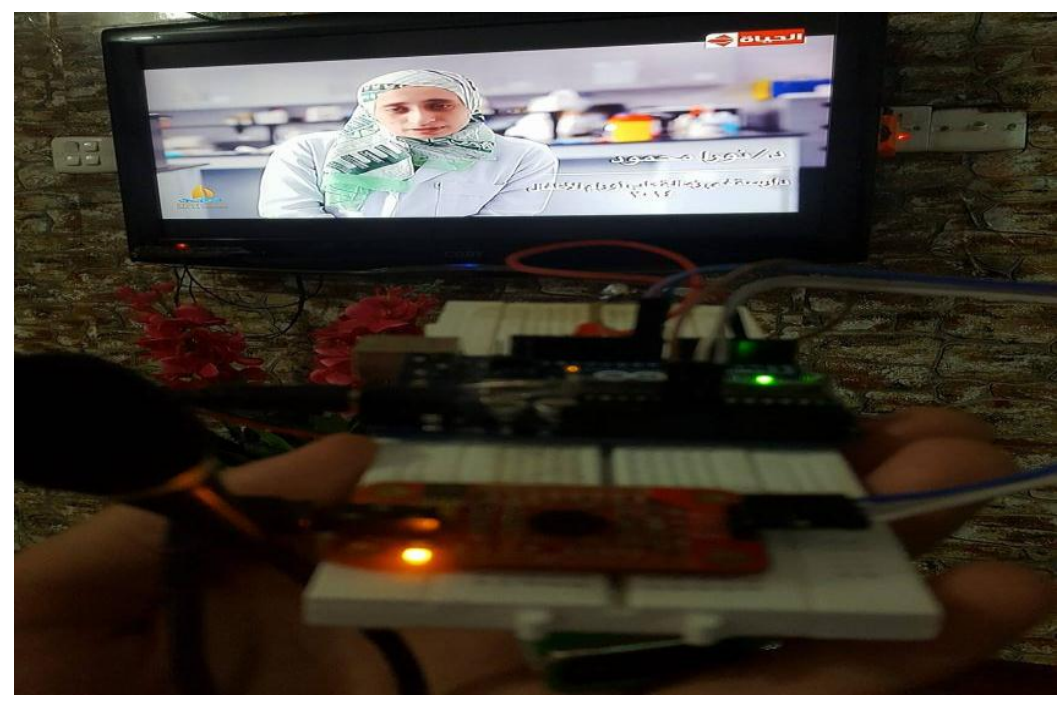

Figure 10: Testing of exist from contents list 


\section{Conclusion}

In this proposed system, suggested approach for controlling home application based on voice command. This system basically targets the elderly and disabled persons to enhance their life in the community. The prototype which has been developed can control the various electrical of a home or office as example in this project is control TV and can be used in any place. It can be concluded this work depending on Arduino microcontroller a successful system. This system consists of an Arduino-Uno board, a voice recognition, IR sensor, IR LED and microphone. It is user sociality and it is cost effective. Low power consumption, it reduces risk. Low cost operating system using Arduino module [ATmega328] in C programming language. Also overall cost effective and parts are easily available on the market.

\section{References:}

[1].Pankaj Bhardwaj, Ashvani Kr Sharma, Meghna Saxena, Saurabh Chaudhari, Arshit Gupta, Home Automation By Voice Recognition Using Zigbee, International Journal of Scientific Research and Management Studies (IJSRMS),(2016)Vol. 2 Issue 12, P.P( 481-486).

[2].Marius Branzila, Constantin Sarmasanu, Gabriel Fanaru, ROBOTVOICE - Voice command of a robot", IEEE, International Conference and Exposition on Electrical and Power Engineering (EPE),(2014).

[3].Sonali Sen, Shamik Chakrabarty, Raghav Toshniwal, Ankita Bhaumik, Design of an Intelligent Voice Controlled Home Automation System", International Journal of Computer Applications, (2015), Vol. 121 No.15, P.P(39-42).

[4].Kharka Bahadur Rai, Jeetendra Thakur, Nirmal Rai, Voice Controlled Wheel Chair Using Arduino, International Journal of Science, Technology \& Management, (2015), Vol.04, Issue. 06,P.P(6-13).

[5].Theodore Ramli, Natashia Nabiha Dabimel, Mazlina Mamat, Norfarariyanti Parimon Rosalyn R. Porle, Simple Speech Controlled Home Automation System Using Android Devices, Journal of Scientific Research and Development (JSRAD), (2016), P.P(3338).

[6].Avadhoot R. Telepatil, Amit N. Hambar, Pushkar S.Terwadkar, Home Automation with MATLAB and ARDUINO Interface, International Journal of Innovative Research in Computer and Communication Engineering, (2017), Vol. 5, Issue 3, P.P (5501-5507).
[7].H.R. Hatem, J. N. Shehab and I. Abdul-Rahman, ARDUINO Microcontroller Based Building, Engineering and Technology Journal, (2017), Vol. 35, Part A, No. 5, P.P(532-536).

[8]. Khaleda Sh. Rejab , Wassan Emad Rauf, Wireless Mobile Robotic Arm Controlled by Ps2 Joystick based on Microcontroller, Diyala Journal of Engineering Sciences, (2017), Vol. 10, No. 3, pp( 4453).

[9].Voice Recognition Module V3 Speak to Control (Arduino compatible), https://github.com/elechouse/VoiceRecognitionV3.gi .

[10]. Mukesh Kumar, Shimi S.L, Voice Recognition Based Home Automation System for Paralyzed People, International Journal of Advanced Research in Electronics and Communication Engineering (IJARECE), (2015) Vol. 4, Issue 10.

[11]. https://learn.sparkfun.com/tutorials/ircommunication\#ir-communication-basics.

[12]. Suha I. Al-nassar, Haraa Raheem Hatem, Jinan N. Shehab, Design and Implementation of Infrared (IR) Communication System, Diyala Journal of Engineering Sciences, (2018), Vol. 11, No. 3, pp( 2933). 